\title{
The role of external cues for endogenous advance reconfiguration in task switching
}

\author{
IRING KOCH \\ Max Planck Institute for Psychological Research, Munich, Germany
}

\begin{abstract}
Most studies of task-set switching rely on cuing paradigms, in which external cues indicate the upcoming task. The present study used an entirely predictable task sequence in a variant of the alternatingruns paradigm of Rogers and Monsell (1995). Preparation effects with purely internal memory cues were compared with those in another experimental group with additional external cues presented prior to the stimulus. External cues led to strongly reduced shift costs with prolonged preparation time. However, this effect was much smaller with internal cues only. To account for this differential effect of preparation time as a function of cue type, it is suggested that internal cues select the next task set, which is sufficient to perform the task. External cues additionally facilitate preparatory retrieval of task-specific stimulus-response rules. This account may also explain why incidental task-sequence learning based on internal cues did not reduce shift costs.
\end{abstract}

The processes underlying task preparation can be investigated in the task-switching paradigm (for a review, see, e.g., Monsell, 1996). Here, typically a condition in which a task is repeated is compared with one with a task switch. The difference between these conditions, both in terms of reaction times (RTs) and error rates, is referred to as shift costs (e.g., Allport, Styles, \& Hsieh, 1994; Gopher, Armony, \& Greenshpan, 2000; Jersild, 1927; Koch, 2001; Mayr \& Keele, 2000; Meiran, 1996; Rogers \& Monsell, 1995; Spector \& Biederman, 1976). The general idea behind shift costs is that they reflect an underlying process that "reconfigures" the cognitive system (i.e., to shift task set) to perform one or the other task, and that this reconfiguration can be achieved prior to the presentation of the stimulus (De Jong, 2000; Gopher et al., 2000; Goschke, 2000; Meiran, 1996; Rogers \& Monsell, 1995; Sohn \& Carlson, 2000). The evidence for this notion is mainly based on studies that vary taskpreparation time.

One major method of varying preparation time is the cuing paradigm (Meiran, 1996). Here, the sequence of tasks is random, but each stimulus is preceded by an external instructional cue for some interval. Indeed, prolonging the cuing interval typically reduces shift costs (see, e.g., Meiran, 1996; Meiran, Chorev, \& Sapir, 2000), suggesting cue-based task reconfiguration.

The author thanks Stefanie Schuch for many discussions, Silvija Mikerevic and Anne Zirngibl for conducting the experiment, and Marina von Bernhardi and Heide John for stylistic suggestions. Thanks also to Rich Carlson, Veronica J. Dark, Ulrich Mayr, Nachshon Meiran, and an anonymous reviewer, who provided valuable comments on previous versions of this article. Correspondence concerning this article should be addressed to I. Koch, Max Planck Institute for Psychological Research, Amalienstrasse 33, D-80799 Munich, Germany (e-mail: koch@ psy.mpg.de).
Task reconfiguration in the cuing paradigm must be triggered by an external task cue, because otherwise the participants would not know which task to perform on the stimulus. Although externally cued reconfiguration may reveal relevant information about task switching processes, it is clearly important to also explore characteristics of reconfiguration triggered by internal cues. The difference between external and internal cues is that an external cue is defined as a current perceptual stimulus, whereas an internal cue is based on memory of previous tasks and future intentions.

A method for examining internally cued reconfiguration was introduced by Rogers and Monsell (1995). In their "alternating runs" paradigm, the presentation of external task cues is logically not required, because participants shifted from one task (A) to the other (B) in a predictable manner: AABBAABB, and so on. Rogers and Monsell used compound stimuli, with one stimulus attribute relevant for the current task, the other relevant for the competing task, so that stimuli were bivalent. Because of the predictable task sequence, there is no need to present instructional cues prior to stimulus onset, as must be done in the cuing paradigm. Therefore, preparation time is varied in the alternating runs paradigm by manipulating the response-stimulus interval (RSI) instead of a cuing interval. In fact, Rogers and Monsell observed that prolonging the RSI reduced shift costs substantially, although not entirely ("residual" costs remained even after very long RSIs). They interpreted this partial reduction of shift costs as evidence for internally cued, "endogenous" advance reconfiguration.

Although the sequence of tasks was entirely predictable, stimuli were also successively presented clockwise in one out of four quadrants on the screen, so that "crossing" either the horizontal or the vertical axis indicated a task shift. That is, the spatial location of the stim- 
ulus served as a "reliable external cue to position in the AABB cycle" (Rogers \& Monsell, 1995, p. 212). This minimized memory requirements to keep track of the current task, because stimulus position unambiguously indicates the task to perform. Therefore, it is likely that the reduction of shift costs was based, at least to some degree, on the perceptually salient clockwise "movement" of the stimulus from one quadrant to the next, so that predicting the next stimulus position and thus the task was considerably aided by the external spatial cue. The goal of the present study was to investigate advance reconfiguration when the intention to switch tasks must be entirely retrieved from memory and is not aided by an additional external cue.

For this goal to be accomplished, the instruction had to focus on the task sequence and external cues had to be omitted. Recently, Kray and Lindenberger (2000) conducted such an experiment, comparing performance across different age groups. They did not provide external cues but still found significantly reduced shift costs with a long RSI, supporting the notion of endogenous advance reconfiguration (see also Goschke, 2000). However, there was no control condition with additional external cues, so that this study does not tell us whether or not internally cued reconfiguration functions in the same way as externally cued reconfiguration. To investigate this issue, the present study compared performance of a group without external cues with that of another group, in which an additional external symbolic cue was provided.

In the present study, participants shifted predictably between two numerical judgment tasks, deciding either whether a given digit was higher or lower than 5 , or whether it was odd or even (see Sudevan \& Taylor, 1987). At the beginning of each block, they were told with which task to start and then to repeat and shift the task in alternation. Within blocks of 12 trials each, RSI was constant, but it varied between blocks. Importantly, in a no-cue group there were no further external cues during this time to aid reconfiguration after the first task was executed. To evaluate the relative benefit of preparation time with internal cues, a second group was tested that was provided with an additional, redundant external cue (cue group). This was accomplished by presenting stimuli in a rectangular frame. In the no-cue group, the shape of the stimulus presentation frame remained constant, whereas its shape covaried with the task in the cue group. Performance in the no-cue group should allow examination of the degree to which internally cued, purely memorybased advance reconfiguration occurs. Furthermore, the comparison with the cue group should indicate the relative benefit when reconfiguration can be (but need not be) based on a redundant, external cue.

\section{METHOD}

\section{Participants}

Thirty-two participants (20 female, 12 male) took part and received $10 \mathrm{DM}$. Their mean age was 27.5 years. Sixteen participants were randomly assigned to each of the two independent groups: nocue and cue.

\section{Stimuli and Tasks}

Stimuli were the digits 1-9, excluding 5. Their height was approximately $0.8 \mathrm{~cm}$. They were centrally presented inside a square or diamond frame measuring $3.8 \times 3.8 \mathrm{~cm}$ on a computer screen (15 in.) connected to an IBM-compatible PC. Stimulus presentation and response registration was synchronized with the vertical refresh rate of the screen. The tasks were to decide whether the digit was odd or even, or whether it was smaller or greater than 5. Stimulus order was random, with the constraint that immediate repetition was not allowed. Responses were made by pressing either the left or the right button of an external response panel. Response keys measured $1.7 \times 1.7 \mathrm{~cm}$ and were separated by $3.3 \mathrm{~cm}$.

\section{Procedure}

The participants were informed that stimuli would be individually presented in a rectangle frame and that responses should be fast but accurate. The participants were then informed that they would have to perform fixed task sequences of 12 trials: AABBAABBAABB. Whether $A$ denoted the odd/even or the greater/smaller task was held constant for each participant but was counterbalanced across participants. They were then given a schema of the S-R mappings for each task, placed beneath the screen. An error message appeared for $500 \mathrm{msec}$ on the bottom part of the screen if they pressed the wrong key.

Before each block started, a written message indicated RSI duration, which was either 200 or 1,000 msec. For both groups, it was strongly emphasized that they use this interval to prepare for the upcoming task. In the no-cue group, the stimulus presentation frame had the shape of a square and remained on the screen, so that there was no external trigger to start reconfiguration. In the cue group, the shape (i.e., square vs. diamond) covaried with the task to provide an external, albeit redundant task cue. Therefore, the response caused the frame to disappear and reappear on the screen after $100 \mathrm{msec}$ in the shape associated with the next task. This was done because otherwise only switch trials would be accompanied by a sudden change in the display (i.e., from square to diamond or vice versa). Due to the short frame removal, the possible exogenous "alerting" effect of this change (see Meiran et al., 2000) should have been the same on switch and nonswitch trials. The participants in the cue group were made aware of the covariation, but the predictable task sequence was emphasized.

After each block of 12 trials, participants received feedback of their mean RT and were encouraged to improve performance. The experiment consisted of 20 blocks (i.e., 240 trials), and blocks with long and short RSI alternated. The participants first received four practice trials (i.e., AABB), for both the long and short interval, to get familiar with the situation. S-R mapping and task in the first trial was counterbalanced across participants. The experiment took about $30 \mathrm{~min}$.

\section{Design}

The experiment tested whether the effect of varying the preparation interval on shift costs differs as a function of whether participants received an additional, redundant task cue or not (i.e., cuebased vs. memory-based preparation). The independent variables were group (cue vs. no cue) as well as RSI (200 vs. 1,000 msec) and trial type (task switch vs. nonswitch) as within-subjects variables. The dependent variables were RT and errors.

\section{RESULTS}

The first two trials of each block were not analyzed because the first task and thus also its repetition were ex- 
ternally cued by the instruction given before each block. Error rates were determined for each participant as a function of trial type and RSI. For the RT analysis, incorrect trials, those following them as well as RTs above $2,500 \mathrm{msec}[1.09 \%$ vs. $0.59 \%$ for the no-cue and cue groups, respectively; $t(30)=1.25, S E=0.39, p>.2]$ were discarded. For the remaining RTs, the median was determined for each participant as a function of trial type and RSI. Table 1 (left side) shows these data as a function of group, trial type, and RSI.

These data were submitted to an analysis of variance (ANOVA) with the independent variables group (cue vs. no cue), RSI (short vs. long), and trial type (switch vs. nonswitch). Significance was tested at $\alpha=.05$. The analysis yielded a significant main effect of trial type $\left[F(1,30)=91.97, M S_{\mathrm{e}}=25,711\right]$, showing that RTs in switch trials were higher than those in nonswitch trials ( $885 \mathrm{vs.} 613 \mathrm{msec}$ ). Also the main effect of RSI was significant $\left[F(1,30)=26.66, M S_{\mathrm{e}}=7,957\right]$, indicating that RTs for the short RSI were higher than for the long RSI $(789$ vs. $708 \mathrm{msec})$. These variables interacted $[F(1,30)=$ $\left.9.14, M S_{\mathrm{e}}=5,441\right]$. Because trial type effects reflect shift costs, the interaction indicates that shift costs were lower with the long RSI.

Furthermore, there was a significant interaction of group and RSI $\left[F(1,30)=16.11, M S_{\mathrm{e}}=7,957\right]$, indicating a stronger RSI effect in the cue group $(137 \mathrm{msec})$ than in the no-cue group (30 msec). Most important, however, is the three-way interaction of group, RSI, and trial type $\left[F(1,30)=5.51, M S_{\mathrm{e}}=5,441\right]$. The reduction of shift costs with long as opposed to short RSIs was much stronger in the cue group $(140 \mathrm{msec})$ than in the no-cue group $(18 \mathrm{msec})$. Neither the main effect of group nor the interaction of group and trial type was significant $\left(F_{\mathrm{S}}<1\right)$.

To test whether the RSI effects in the no-cue group were significant, the data of this group were analyzed separately with the independent variables trial type and RSI. This analysis revealed a significant main effect of trial type $\left[F(1,15)=44.43, M S_{\mathrm{e}}=29,599\right]$, but both the main effect of RSI and the interaction of trial type and RSI were not significant $\left(F_{\mathrm{S}}<1\right)$. Hence, although participants in the no-cue group were clearly able to reconfigure for task shifts, they did not benefit from a longer preparation time. In contrast, the same analysis for the cue group yielded highly significant main effects for RSI $\left[F(1,15)=38.73, M S_{\mathrm{e}}=8,653\right]$ and for trial type $\left[F(1,15)=48.41, M S_{\mathrm{e}}=21,823\right]$, as well as a highly significant interaction $\left[F(1,15)=10.57, M S_{\mathrm{e}}=7,421\right]$.

Given this pattern of RT results, analysis of error rates is important in order to exclude the possibility that the reason participants in the no-cue group did not use the RSI for reconfiguration was that they "got lost" in the task sequence. Although the fact that accuracy was $94 \%$ overall in that group clearly speaks against this possibility, error rates (see Table 1, right panel) were submitted to the same analysis as were RTs. Neither the main effect of group nor any interaction with this variable was significant $\left(F_{\mathrm{S}}<1\right)$, suggesting that the conditions in the nocue group were not fundamentally more difficult than those of the cue group. Also, the number of blocks with more than one error did not differ significantly between groups [mean number was 3.06 for the no-cue group and 2.31 for the cue group, $t(30)=.59, S E=1.28, p>.56]$. The only significant effects in the ANOVA were the main effects of trial type $\left[F(1,30)=16.29, M S_{\mathrm{e}}=.0009145\right]$ and RSI $\left[F(1,30)=6.93, M S_{\mathrm{e}}=.001078\right]$, with all other $F_{\mathrm{S}}<1$. Error rates were higher in switch trials than in nonswitch trials ( $6.6 \%$ vs. $4.4 \%$ ), but, unexpectedly, error rates were higher with long than with short RSIs $(6.3 \%$ vs. $4.8 \%$ ). This suggests that participants lowered their response thresholds in the long RSI condition, which led to faster responses but more errors. Therefore, the RSI main effect in the RT data might be due partly to a speed-accuracy tradeoff. Importantly, however, this tradeoff could not explain the significant interactions in the RT data.

\section{DISCUSSION}

The aim of the present study was to examine "purely" memory-based reconfiguration in the alternating-runs procedure of Rogers and Monsell (1995). This was done by comparing a "no-cue" group with a cue group in which reconfiguration was aided by a redundant external cue. The cue group showed a substantially stronger reduction of both RT level and shift costs with prolonged preparation time (RSI) as compared with the no-cue group.

Table 1

Mean Reaction Time (RT, in Milliseconds) and Error Percentage (With Standard Errors) as a Function of Trial Type (Switch vs. Nonswitch), Response-Stimulus Interval (RSI, Long vs. Short), and Presence of Task Cue (No Cue vs. Cue)

\begin{tabular}{|c|c|c|c|c|c|c|c|c|c|c|}
\hline \multirow[b]{2}{*}{ Condition } & \multicolumn{2}{|c|}{ Switch } & \multicolumn{2}{|c|}{ Nonswitch } & \multirow[b]{2}{*}{ Shift Cost } & \multicolumn{2}{|c|}{ Switch } & \multicolumn{2}{|c|}{ Nonswitch } & \multirow[b]{2}{*}{ Shift Cost } \\
\hline & RT & $\overline{S E}$ & $\mathrm{RT}$ & $\overline{S E}$ & & $\% \mathrm{E}$ & $\overline{S E}$ & $\% \mathrm{E}$ & $\overline{S E}$ & \\
\hline \multicolumn{11}{|l|}{ No Cue } \\
\hline Short RSI & 915 & 57 & 620 & 32 & 295 & 6.6 & 2.2 & 4.3 & 1.4 & 2.3 \\
\hline Long RSI & 888 & 72 & 611 & 36 & 277 & 7.4 & 1.7 & 6.1 & 2.1 & 1.3 \\
\hline \multicolumn{11}{|l|}{ Cue } \\
\hline Short RSI & 975 & 71 & 648 & 50 & 327 & 5.5 & 1.4 & 2.6 & 1.3 & 2.9 \\
\hline Long RSI & 760 & 58 & 573 & 31 & 187 & 6.8 & 1.8 & 4.8 & 1.4 & 2.0 \\
\hline
\end{tabular}

Note-Short RSI = $200 \mathrm{msec}$, long RSI = 1,000 $\mathrm{msec}$. 
At first sight, the absence of an RSI effect on shift costs in the no-cue group appears to be inconsistent with Kray and Lindenberger's (2000) finding of such an effect. However, when the first two trials in each block were included in the present analysis (as they were in Kray \& Lindenberger's study), the nonsignificant $18-\mathrm{msec}$ RSI effect on shift costs turned into a significant 46-msec effect. Performance in these first trials thus seemed to be influenced by external task instructions between blocks, at least in the present study. Note, however, that Kray and Lindenberger's RSI effect on shift costs was substantially stronger than the present one, presumably because of methodological differences that may have favored internally cued reconfiguration processes. Given these considerations, the crucial result of the present study is the significant group difference in the RSI effects rather than the relatively weak advance reconfiguration effect in the no-cue group.

The present finding that internal cues, in comparison with external cues, result in a much weaker preparation benefit is also supported by recent studies of Heuer, Schmidtke, and Kleinsorge (2001) as well as Koch (2001). They independently found that incidental learning of a task sequence did not reduce shift costs but only general RT level. This further underlines that internally generated cues based on task sequence are comparably ineffective relative to external cues.

Regarding the RSI effect on shift costs with external cues in the cue group, one might object that RSI variations may not be optimal for showing advance reconfiguration (Meiran, 1996; Meiran et al., 2000), because a longer RSI provides more time not only for active task preparation but also for passive dissipation of interference from previous task sets (see also Koch, 2001). However, if passive dissipation played a major role for the present results, it should also affect RTs in the nocue group; but only a minor influence of the RSI was found in that group. It may thus be concluded that advance reconfiguration in the cue group was primarily due to an active process, although a small contribution of passive dissipation cannot be excluded. The crucial point in this context, however, is that advance reconfiguration was much more efficient in the cue group than in the nocue group.

It could be argued that the frame offset and onset in the cue group, together with the sudden change in shape, acted like a "warning signal" to produce alertness in the participants. This frame onset could have increased participants' responsiveness (see Meiran et al., 2000), unlike the frame that remained on the screen in the no-cue group. However, if that had been the case, participants in the cue group ought to have been faster even at the short RSI. Yet if anything, they were slower. When Rogers and Monsell (1995, Experiment 5) presented a neutral warning signal prior to stimulus onset, they found that it reduced RT level slightly but did not modulate shift costs. Also Meiran et al. (2000, Experiment 4) observed that a sharp change in the display during the preparation inter- val led only to RT facilitation, not to reduced shift costs. Therefore, it is unlikely that this task-unspecific alerting factor could account for the stronger reduction of the shift costs with long preparation interval in the cue group as opposed to the no-cue group. Rather, this effect may have been due to the specific information inherent in the frame (i.e., its shape) that cued the next task, suggesting that some part of Rogers and Monsell's (1995) advance reconfiguration effect was related to the external task cues in their paradigm.

To account for the differential effects of internal and external cues in task switching, one has to consider what can be done to prepare a task prior to stimulus presentation. One component of task preparation is to select the relevant task by updating a task "goal" in working memory (see Fagot, 1994; Mayr \& Kliegl, 2000; Rubinstein, Meyer, \& Evans, 2001). This updating process can be based on either external or internal cues. In fact, the high accuracy level in the no-cue group suggests that task selection is not a major problem with internal cues. Possibly, however, task selection is slightly faster with external than with internal cues.

A second component of task preparation is to retrieve the new task-specific S-R rules (Mayr \& Kliegl, 2000). It is likely that external cues are more salient than internal cues, so that they might also be more effective in triggering S-R retrieval prior to stimulus onset. This cue salience account could explain the advance reconfiguration effect with external cues. Furthermore, it could also explain the finding that incidental task-sequence learning did not reduce shift costs (see Koch, 2001). This would be the case if the internal, sequence-based cue mainly primed task selection but not (or only slightly) advance retrieval of the new S-R rules. It appears that internal cues, whether incidentally acquired ("implicit") task predictions or explicitly instructed internal cues, are quite ineffective at initiating the $\mathrm{S}-\mathrm{R}$ retrieval process.

The fact that substantial "residual" shift costs remain even with salient external cues (more than $180 \mathrm{msec}$ in the cue group) shows that advance reconfiguration is limited. This suggests that a part of shift costs is due to stimulus-based interference that cannot be resolved in advance. To account for similar findings, Rogers and Monsell (1995) proposed that stimuli prime not only the current task but also any previous task(s) associated to them. The idea of stimulus priming in task switching has been supported by several recent studies (Allport \& Wylie, 2000; Koch \& Allport, 2003; Waszak, Hommel, \& Allport, in press). Also, there is evidence that stimulusrelated processes (such as response selection) trigger inhibition of competing tasks (or S-R rules; cf. Schuch \& Koch, 2003). It might well be that not only stimuli but also external cues, through their association to the relevant task, help to inhibit competing tasks (cf. Mayr \& Keele, 2000; Schuch \& Koch, 2003). Together, these considerations make clear that task preparation is affected by a variety of internal and external constraints that all contribute to shift costs (Koch \& Allport, 2003). 


\section{REFERENCES}

Allport, A., Styles, E. A., \& Hsieh, S. (1994). Shifting intentional set: Exploring the dynamic control of tasks. In C. Umiltà \& M. Moscovitch (Eds.), Attention and performance XV: Conscious and nonconscious information processing (pp. 421-452). Cambridge, MA: MIT Press.

Allport, A., \& Wylie, G. (2000). Selection-for-action in competing (Stroop) tasks: "Task-switching," stimulus-response bindings, and negative priming. In S. Monsell \& J. S. Driver (Eds.), Attention and performance XVIII: Control of cognitive processes (pp. 35-70). Cambridge, MA: MIT Press.

De JoNG, R. (2000). An intention-activation account of residual switch costs. In S. Monsell \& J. Driver (Eds.), Attention and performance XVIII: Control of cognitive processes (pp. 357-376). Cambridge, MA: MIT Press.

FAGOT, C. (1994). Chronometric investigations of task switching. Unpublished doctoral dissertation, University of California, San Diego.

Gopher, D., Armony, L., \& Greenshran, Y. (2000). Switching tasks and attention policies. Journal of Experimental Psychology: General, 129, 308-339.

Goschke, T. (2000). Intentional reconfiguration and involuntary persistence in task-set switching. In S. Monsell \& J. Driver (Eds.), Attention and performance XVIII: Control of cognitive processes (pp. 333-355). Cambridge, MA: MIT Press.

Heuer, H., Schmidt ke, V., \& Kleinsorge, T. (2001). Implicit learning of sequences of tasks. Journal of Experimental Psychology: Learning, Memory, \& Cognition, 27, 967-983.

Jersild, A. T. (1927). Mental set and shift. Archives of Psychology (Whole No. 89).

Kосн, I. (2001). Automatic and intentional activation of task sets. Journal of Experimental Psychology: Learning, Memory, \& Cognition, 27, 1474-1486.

Koch, I., \& Allport, A. (2003). Cue-based and stimulus-based priming of tasks in task switching. Manuscript submitted for publication.

Kray, J., \& Lindenberger, U. (2000). Adult age differences in task switching. Psychology \& Aging, 15, 126-147.

MAYr, U., \& KeELE, S. W. (2000). Changing internal constraints on ac- tion: The role of backward inhibition. Journal of Experimental Psychology: General, 129, 4-26.

Mayr, U., \& KLIEgL, R. (2000). Task-set switching and long-term memory retrieval. Journal of Experimental Psychology: Learning, Memory, \& Cognition, 26, 1124-1140.

MeIRAN, N. (1996). Reconfiguration of processing mode prior to task performance. Journal of Experimental Psychology: Learning, Memory, \& Cognition, 22, 1423-1442.

Meiran, N., Chorev,Z., \& Sapir, A. (2000). Component processes in task switching. Cognitive Psychology, 41, 211-253.

Monsell, S. (1996). Control of mental processes. In V. Bruce (Ed.), Unsolved mysteries of the mind: Tutorial essays in cognition (pp. 93148). Hove, U.K.: Erlbaum.

Rogers, R. D., \& Monsell, S. (1995). Costs of a predictable switch between simple cognitive tasks. Journal of Experimental Psychology: General, 124, 207-231.

Rubinstein, J., Meyer, D. E., \& Evans, J. E. (2001). Executive control of cognitive processes in task switching. Journal of Experimental Psychology: Human Perception \& Performance, 27, 763-797.

Schuch, S., \& КосH, I. (2003). The role of response selection for inhibition of task sets in task shifting. Journal of Experimental Psychology: Human Perception \& Performance, 29, 92-105.

SoHN, M.-H., \& CARLSON, R. A. (2000). Effects of repetition and foreknowledge in task-set reconfiguration. Journal of Experimental Psychology: Learning, Memory, \& Cognition, 26, 1445-1460.

Spector, A., \& Biederman, I. (1976). Mental set and mental shift revisited. American Journal of Psychology, 89, 669-679.

Sudevan, P., \& TAYlor, D. A. (1987). The cuing and priming of cognitive operations. Journal of Experimental Psychology: Human Perception \& Performance, 13, 89-103.

Waszak, F., Hommel, B., \& Allport, A. (in press). Task-switching and long-term priming: Role of episodic S-R bindings in task-shift costs. Cognitive Psychology.

(Manuscript received September 12, 2000; revision accepted for publication March 11, 2002.) 